\title{
Incertidumbre y riesgo en los cobros desde el punto penal: un análisis aplicando la teoría de juegos*
}

\section{Uncertainty and risk in penalty kicks: an analysis by applying the game theory}

\author{
Alexander Cotte Poveda** \\ Julián Alberto Gutiérrez López****
}

Recibido: 23 de junio de 2015

Revisado: 6 de julio de 2015

Aprobado: 2 de febrero de 2016

\section{Resumen}

La incertidumbre y el riesgo juegan un papel preponderante en el momento de tomar la decisión de cobrar un tiro desde el punto penal. Las implicaciones de ganar un torneo de fútbol de alta competencia tienen efectos de señalización sobre los agentes que participan en los diferentes mercados. Este trabajo de investigación advierte que los cobros desde el punto penal no son simples cuestiones de azar y suerte, son interacciones que deben tener en cuenta, entre muchas otras variables, la técnica, la preparación, las dotaciones de los participantes y que deben ser una combinación de estrategias y probabilidades. Aplicando la teoría de juegos para los tres equipos más representativos de la liga de fútbol de España - Real Madrid, Barcelona y el Atlético de Madrid- y

\footnotetext{
* Este artículo hace parte del programa de investigación desarrollado al interior del grupo Economía y Humanismo y cuenta con la financiación de FODEIN. Los autores agradecen los valiosos y acertados comentarios de los asistentes al XII Simposio Nacional de Microeconomía.

** Universidad Santo Tomás, Facultad de Economía, alexcotte@yahoo.com

*** Escuela Colombiana de Ingeniería Julio Garavito, Facultad de Economía, julkeled@hotmail.com
} 
utilizando los datos de la Primera División de España, Copa del Rey, Mundial de Clubes y la Champions League, todas estas competiciones correspondientes a la temporada 2015-2016 y a la temporada 2014-2015, se determinan las distintas estrategias y probabilidades que el cobrador debe dilucidar para anotar el gol en un cobro desde el punto penal y, con ello, contrarrestar las diferentes probabilidades y estrategias de las que dispone el arquero para atajar el lanzamiento.

Palabras clave: Microeconomía, teoría de juegos, incertidumbre y riesgo, modelos de programación.

Clasificación JEL: D81, C70, C61

\section{Abstract}

Uncertainty and risk play a leading role in the moment of taking the decision to kick from a penalty mark. The implications of winning a highly competitive soccer tournament has signaling effects on the agents involved in the different markets. This research paper warns that kicks from a penalty mark are not mere matters of chance and luck, they are interactions that must take into account, among other variables, the technique, the preparation, the endowments of participants and should be a combination of strategies and probabilities. Applying the game theory to the three most representative teams of the Spanish Soccer League - Real Madrid, Barcelona and Atletico Madrid- and using data from the First Division of Spain, Copa del Rey, Club World Cup and the Champions League, all these competitions corresponding to the 2015-2016 season and 2014-2015 season, are determined the various strategies and probabilities that the kicker of the penalty must elucidate to score a goal from the penalty mark and, thus, counteract the different probabilities and strategies the goalkeeper has stop the ball.

Keywords: Microeconomics, game theory, uncertainty and risk programming models.

Classification JEL: D10, D20 


\section{Introducción}

En las últimas décadas, el deporte ha suscitado un enorme interés en el público en general. Particularmente, la predilección hacia el fútbol ha logrado que muchas de las preferencias se inclinen y se interesen por entender en qué consiste este juego y saber el porqué de su atrayente relación entre sus diversos aficionados. La investigación relativamente reciente, en especial, el uso de la teoría de juegos y la estadística, es una muestra de la relevancia del tema y sus implicaciones. Desde hace unos años fútbol dejó de ser simplemente un deporte para convertirse en lo que conocemos actualmente. Hace más de treinta años este deporte era rudimentario, tanto en lo relacionado con la dotación de los jugadores como con el diseño de las estrategias, pero a lo largo de los años, la inclusión de la tecnología y la inversión en términos de publicidad han hecho que este deporte se transforme e integre distintas disciplinas en sus desarrollos. Hoy en día es bastante común, ver en los entrenamientos no solo al personal propio del área del deporte, como nutricionistas y psicólogos, sino que también podemos encontrar estadísticos y economistas, todo con el fin de no dejar nada a los que algunos llaman el "azar".

La evolución reciente del deporte, las aplicaciones acontecidas en el fútbol y, específicamente, lo relacionado con los cobros desde el punto penal en el fútbol sugieren la aplicación de la teoría económica para tratar de determinar cómo se puede reducir la incertidumbre y el nivel de riesgo en la interacción estratégica de los diferentes agentes participantes en el juego. Esto supone que se deben presentar una serie de circunstancias en las que, en algún momento determinado, se determine una condición estratégica ideal tanto por su sencillez como por su aplicación práctica, la presencia sin igual de los diferentes incentivos económicos que se ven implicados en la ejecución de los diferentes lanzamientos, este es un estado ideal para testear los razonamientos fundamentales de la teoría de juegos.

Generalmente, la prensa especializada argumenta que la definición del lanzamiento desde el punto penal es una cuestión de suerte con una gran proporción de situaciones azarosas. Esta idea nos lleva a formular una pregunta central que para el común de la gente ha sido un juicio de valor poco debatido: ¿serán los penales una lotería? Claramente, los jugadores no calculan cuál es la mejor estrategia para marcar el gol en su lanzamiento ni cuál(es) es o son la(s) estrategia(s) del portero para evitar la anotación. La incorporación de expertos de las diferentes áreas del conocimiento ha logrado el diseño de una serie de estrategias que determinan la mejor alternativa probable que cada jugador deberá adoptar, convirtiendo esta "lotería" en una interacción estratégica entre los participantes, interacción en la que, por supuesto, se debe incluir que la mejor estrategia posible depende de lo que piense que va a hacer el otro.

En este contexto se trata de indagar si los participantes del juego, es decir, los jugadores, compiten para lograr el mejor resultado posible utilizando la mejor estrategia óptima 
y, por consiguiente, si persiguen la senda de la mejor alternativa dadas las predicciones analizadas y determinadas por el equilibrio de Nash (1950), criterio imprescindible en la teoría económica que es uno de los sustentos principales de la teoría de juegos.

Con este propósito, el artículo se ha organizado en cinco secciones. La primera está constituida por la presente introducción. La segunda parte expone el marco teórico conceptual en el que está fundamentado el trabajo de investigación. El tercer acápite describe una breve revisión de la literatura. La cuarta sección presenta la aproximación metodológica, y en ella se discuten los principales resultados. Finalmente, en la última parte se exponen las conclusiones de la investigación.

\section{Marco teórico conceptual}

La teoría económica de juegos que soportaría la aproximación a los lanzamientos desde el punto penal en el juego del fútbol se conoce en la teoría de juegos como las estrategias mixtas (Chiappori, Levitt, y Groseclose, 2002), en este trabajo, se analizan los penaltis en el fútbol como una aplicación general para el análisis de las estrategias mixtas. Esta aplicación plantea que el jugador, al momento de lanzar el cobro desde el punto penal, no solo tiene una estrategia, sino un conjunto de alternativas posibles mediante la elección aleatoria de diversas y potenciales estrategias; estas diferentes posibles decisiones estratégicas se pueden determinar por medio de la implementación de una estrategia mixta a partir de estrategias puras. La característica singular de una estrategia mixta radica en que la toma de decisiones se caracteriza por estar en presencia de condiciones de riesgo e incertidumbre; para el cobro de los lanzamientos es un estado emocional en el que los participantes se enfrentan a una serie de incentivos. En presencia de una denominada estrategia pura, la mejor alternativa posible se decide con certeza.

En la teoría de juegos con estrategias mixtas, se plantea que el jugador, al momento de lanzar el cobro, no solo tiene una estrategia, sino, como lo afirma Palacios-Huerta (2003) cuando explica la estrategia general, "lo que hace es asignar una probabilidad a cada una de ellas. Resulta útil pensar que los jugadores lo que eligen es una regla para seleccionar de manera aleatoria entre sus estrategias originales". Desde esta perspectiva, y con las ideas explicadas por Kreps (1990), al existir $\sum_{i}$ como el conjunto de las diferentes distribuciones de una probabilidad sobre $\mathrm{Si}$, un elemento $\sigma_{i} \in \sum_{i}$ se denomina una estrategia mixta para el jugador $i$, así, los juegos con presencia de estrategias mixtas son distribuciones de probabilidad sobre el conjunto de estrategias puras, de manera formal (Gibbons, 1992), en el juego de la forma $G=\left(j, s_{p}, \ldots, s_{n}: u_{p}, \ldots, u_{n}\right)$, y determinadas las diferentes estrategias puras de los jugadores mediante el conjunto $s_{i}=\left\{s_{i p}, \ldots, s_{i k}\right\}$. Contrario al enfoque ex ante, la existencia del equilibrio no depende de la racionalización de las estrategias, sino de la consistencia ex post de estas, es decir, de que los jugadores pueden observar cada vez que se enfrentan a esa situación cómo se obtienen los mejores resultados posibles, por lo cual, ningún jugador tiene razones para modificar su 
estrategia, convirtiendo este equilibrio en un equilibrio estático. Se especifica una denominada estrategia mixta para la distribución de probabilidades para el jugador $i$ sobre $s_{i}$ mediante la siguiente especificación:

$$
\pi_{i}=\left(\pi_{i 1}, \ldots, \pi_{i k}\right), \quad \sum_{k} \pi_{i k}=1 ;(1)
$$

La expresión $\pi_{i k} \equiv \operatorname{Pr}\left\{s_{i}=s_{i k}\right\}$ representa cada uno de los elementos $\sigma_{i}$ para la probabilidad de que el participante en el juego $i$ establezca su estrategia, que debe ser pura en $s_{k}$. En el anterior contexto, los jugadores participantes deben optar por la estrategia mixta más adecuada. $\mathrm{Al}$ incluir la posibilidad de elección de estrategias, $\sigma$ no estaría asociado con el desarrollo de un solo juego, sino con el de varios, y cada uno con una probabilidad asociada, con esto la ganancia que espera lograr el jugador i cuando cada uno de los jugadores elige una estrategia mixta $\sigma_{i}$ se define así:

$$
V_{i}(\pi)=\sum_{s}\left[\prod_{j} P_{\pi}^{j}(s) U_{i}(s)\right](2)
$$

Se observa que la ganancia que el jugador $i$ espera obtener se presenta como la sumatoria ponderada de las ganancias agrupadas en cada una de las combinaciones de las estrategias puras s. De manera general, un juego se puede representar de la forma $G=\left(s_{1}, \ldots, s_{n} ; u_{1}, \ldots, u_{n}\right)$, espacios de estrategias para los jugadores $\mathrm{S}_{1}, \ldots, \mathrm{S}_{\mathrm{n}} \mathrm{y}$ sus respectivas funciones de ganancias $u_{1}, \ldots u_{n}$. Las estrategias mixtas cumplen con la relación $\pi^{*}=\left(\pi_{1}^{*}, \ldots, \pi_{i}^{*}, \ldots, \pi_{n}^{*}\right)$ que representa un equilibrio de Nash si para cada jugador $\mathrm{i}$ :

$$
U_{i}\left(\pi_{1}^{*}, \ldots, \pi_{i-1}^{*}, \pi_{i}^{*}, \pi_{i+1}^{*}, \ldots, \pi_{n}^{*}\right) \geq U_{i}\left(\pi_{1}^{*}, \ldots, \pi_{i-1}^{*}, \pi_{i}, \pi_{i+1}^{*}, \ldots, \pi_{n}^{*}\right) \forall \pi_{i} \operatorname{de} \Delta\left(s_{i}\right)(3)
$$

Para cada uno de los jugadores representados por i, $\pi_{-} i^{\wedge *}$, es una posible solución con la estructura de maximización (Kreps, 1990):

$$
\text { Máx } U_{i}\left(\pi_{1}^{*}, \ldots, \pi_{i-1}^{*}, \pi_{i}, \pi_{i+1}^{*}, \ldots, \pi_{n}^{*}\right)(4)
$$

En esta relación, $\pi_{i} \in \Delta\left(s_{i}\right)$.

La literatura sobre teoría de juegos afirma que las estrategias mixtas son una generalización de las estrategias puras y que, como tal, son una lotería de las estrategias puras para determinar un resultado coherente para cada estrategia definida dentro de un marco de espacios de estrategias, ganancias, probabilidades y valores esperados.

\section{Revisión de la literatura}

Los antecedentes de investigación revelan la escasez de trabajos sobre el tema indagado utilizando la teoría de juegos y su aplicación. La literatura relativamente reciente muestra una variedad de alternativas para analizar. Existen diversos planteamientos, enfoques y aproximaciones por parte de los diferentes autores. 
Chiappori, Levitt y Groseclose (2002) utilizan el concepto de estrategia mixta como un elemento fundamental de la teoría de juegos para estudiar la ejecución de los penaltis en el fútbol como una aplicación en el análisis de las estrategias mixtas. Implementan un modelo de probabilidades para los participantes del juego y por medio de la evidencia empírica prueban las hipótesis y predicciones del modelo utilizando un conjunto de datos de 459 penaltis para las ligas europeas de Italia y Francia. El trabajo concluye afirmando que los resultados empíricos son consistentes con las predicciones del modelo propuesto, por ende, no se puede rechazar la hipótesis según la cual los jugadores no seleccionan de forma óptima sus estrategias condicionando el comportamiento del oponente. Finalmente, se advierte que es importante tener en cuenta la heterogeneidad de los jugadores, ya que desempeña un papel crítico en el análisis; los autores argumentan que la presencia de dicha heterogeneidad tiene implicaciones en los resultados.

Palacios-Huerta (2003) muestra las repercusiones del teorema del Minimax en un juego de suma cero, es decir, que en el lanzamiento de un cobro desde el punto penal esta acción se determina como un juego de suma cero, ya que si el cobrador anota el penalti gana y el arquero pierde, pero si el cobrador no acierta, este es el que pierde en la solución propuesta. El trabajo ofrece un análisis empírico de un aspecto de la teoría de las interacciones estratégicas, una aplicación del concepto fundamental en muchas situaciones estratégicas en las que las acciones de uno no deben ser predecibles por el adversario en el caso práctico del cobrador y del que debe impedir la anotación. El trabajo aplica desde este contexto la teoría de juegos con estrategia mixta, incluye el teorema Minimax de von Neumann y la noción general del equilibrio de Nash en situaciones estratégicas que requieren imprevisibilidad. Para el análisis empírico se utilizan los datos provenientes de las ligas profesionales de Italia, España e Inglaterra para el periodo comprendido desde septiembre de 1995 hasta junio de 2000. Se concluye que, como en todos los juegos estrictamente competitivos, las preferencias de los jugadores son diametralmente opuestas en un penal; igualmente, se afirma que los resultados originados por la evidencia son consistentes con el juego y su equilibrio en los aspectos teóricos más relevantes, los jugadores generan en serie secuencias independientes e ignoran los posibles vínculos estratégicos entre los jugadores.

Apesteguia y Palacios-Huerta (2010) argumentan que existen ciertos estados de la naturaleza que inciden en la toma de decisiones, entre ellos los relacionados con las emociones. En esta perspectiva, la naturaleza rara vez crea condiciones que permiten una visión transparente de elementos psicológicos en la ejecución de una labor determinada, pero cuando lo hace, los fenómenos suelen ser demasiado complejos como para discernir claramente el impacto de estos elementos en la conducta humana. Utilizando un experimento asociado con profesionales que tienen que realizar una tarea simple en una competición en un torneo y usando como caso especial el fútbol, los autores aclaran que, para definir el ganador de un partido, en algún momento de la competición se 
necesita definirlo desde la ejecución de tiros desde el punto penal; para ello utilizan una muestra de datos de 1343 penales de 129 tandas durante el período 1976-2003. Usando los modelos de efectos aleatorios y un modelo de datos paneles dinámicos, muestran las probabilidades y el mecanismo que permita dilucidar por qué el equipo que inicia los lanzamientos tiene una probabilidad más alta de ganar. Una de las conclusiones más sobresalientes del trabajo confirma la idea de que el equipo que inicie la ejecución de los cobros tiene una probabilidad de ganar del $60.5 \%$.

Desde la teoría de la economía experimental, Palacios-Huerta y Volij (2008) realizan un experimento convencional en el que los sujetos juegan un juego cuyo único equilibrio implica estrategias mixtas. Centrándose en el fútbol, argumentan que este deporte tiene una serie de características únicas que lo hacen especialmente adecuado para aplicar el experimento, por ejemplo, jugadores profesionales que se enfrentan a una sencilla interacción estratégica que se rige por normas muy detalladas y que se ponen a prueba en la ejecución de un penal. Esta estructura formal de la interacción es fácilmente reproducible en el laboratorio, y la evidencia precedente de investigaciones ha encontrado que los jugadores de fútbol profesionales muestran que su comportamiento es consistente con el equilibrio que predice la teoría de juegos en presencia de juegos de suma cero y estrategias mixtas. Los autores concluyen que no se puede descartar la idea de que años de experiencia de campo en diferentes situaciones estratégicas de suma cero, no solo en el análisis de los penales, han tenido efectos en los jugadores de fútbol profesional, efectos relacionados con un estado emocional donde ellos esperan que su cobro no sea adivinado.

Palacios-Huerta (2014) realiza una compilación de investigaciones desde el enfoque de la economía en la que el fútbol es uno de los principales temas de investigación. Desde la perspectiva de la economía, se argumenta que se pueden analizar los deportes y, por supuesto, dentro de este gran conjunto el tema del fútbol se ha convertido en los últimos tiempos en un área de investigación. Los resultados más recientes de la investigación económica han evidenciado que la ciencia económica puede explicar muchos de los temas que sobre el fútbol se discuten, pero no solo sobre este deporte, sino también de otros. La idea central del documento es reflexionar acerca de los posibles aportes que el fútbol le puede sugerir a la economía.

La revisión de la literatura permite determinar que son muy pocos los trabajos que, con la aplicación de la teoría de juegos, intentan implementar los desarrollos de la teoría en un entorno particular, en un contexto de incertidumbre y riesgo focalizado, en este caso, en el tema del fútbol, específicamente en los lanzamientos desde el punto penal.

\section{Aproximación metodológica y estrategia empírica}

La estrategia metodológica consiste en implementar una estructura que tenga en cuenta los juegos de suma cero con estrategias mixtas con el apoyo de la inducción retrospectiva. 
El análisis de la información se realizó a partir de los partidos que se jugaron durante diferentes torneos, a saber: Liga de España, Copa del Rey, Champions League y Mundial de Clubes correspondientes al periodo comprendido a los años 2014-2015 y 2015-2016. Se aplica la teoría de juegos para los tres equipos más representativos de la liga de futbol de España: Barcelona, Real Madrid y el Atlético de Madrid. La recolección de la información se formalizó por medio de la revisión visual de datos en medios digitales, y esta fue contrastada con la información obtenida de las bases de datos de las diferentes ligas y torneos. Se analizaron, fecha a fecha, los juegos en cuyos desarrollos de las diferentes jugadas se sancionaron ejecuciones desde el punto penal.

Con la implementación de la teoría de juegos, el objetivo principal es intentar determinar la mejor de las soluciones posibles al dilema que se genera en la toma de decisiones al momento de ejecutar el lanzamiento desde punto penal por parte del cobrador, así como también disminuir el nivel de incertidumbre del portero para la elección del lugar al que se debe arrojar para evitar la anotación. Con esta lógica se pretende determinar las estrategias más eficientes para los participantes en el juego.

Para el caso del jugador denominado cobrador de las ejecuciones desde el punto penal, se le asignaron varias posibles estrategias del lanzamiento, diferenciando primero las estrategias abajo, arriba y medio, y luego las estrategias izquierda, derecha y centro y finalmente la combinación de las diferentes estrategias asignadas (ver tabla 1).

Tabla 1. Resultados de los cobradores

\begin{tabular}{|l|c|c|c|c|}
\hline & Goles & Fallos & Total & \% Gol \\
\hline Abajo & 38 & 8 & 46 & $82,6 \%$ \\
\hline Medio & 9 & 9 & 18 & $50,0 \%$ \\
\hline Arriba & 10 & 4 & 14 & $71,4 \%$ \\
\hline-------- & - & --------- & ---------- \\
\hline Izquierda & --------- & 29 & $69,0 \%$ \\
\hline Centro & 20 & 9 & 19 & $68,4 \%$ \\
\hline Derecha & 13 & 6 & 30 & $80,0 \%$ \\
\hline
\end{tabular}

Dadas las diferentes combinaciones de las estrategias posibles para el cobrador al momento de realizar el cobro desde el punto penal, los resultados originan la matriz de posibilidades expuesta en la tabla 2. 
La responsabilidad institucional pública: un análisis para el mejoramiento de la gestión y la gerencia...

Clara Inés González Sotelo,.Gloria Stella Naranjo Africano

Tabla 2. Resultados de los cobradores en combinación de estrategias

\begin{tabular}{|c|c|c|c|c|}
\hline & Goles & Fallos & Total & $\%$ Gol \\
\hline Izquierda-Arriba & 1 & 1 & 2 & $50,0 \%$ \\
\hline Izquierda-Centro & 4 & 4 & 8 & $50,0 \%$ \\
\hline Izquierda-Abajo & 15 & 4 & 19 & $78,9 \%$ \\
\hline Centro-Arriba & 5 & 2 & 7 & $71,4 \%$ \\
\hline Centro-Medio & 2 & 3 & 5 & $40,0 \%$ \\
\hline Centro-Abajo & 6 & 1 & 7 & $85,7 \%$ \\
\hline Derecha-Arriba & 5 & 1 & 6 & $83,3 \%$ \\
\hline Derecha-Medio & 3 & 2 & 5 & $60,0 \%$ \\
\hline Derecha-Abajo & 16 & 3 & 19 & $84,2 \%$ \\
\hline
\end{tabular}

Fuente: Elaboración propia.

Para el caso del jugador denominado arquero, se le asignaron varias posibles estrategias con el objetivo de detener el lanzamiento, estableciendo, en primera instancia, las estrategias abajo, arriba y medio; posteriormente, las estrategias izquierda, derecha y centro tabla 3; y finalmente, una combinación de estrategias.

Tabla 3. Resultados de los arqueros

\begin{tabular}{|c|c|c|c|c|}
\hline & Goles & Fallos & Total & \% Gol \\
\hline Abajo & 46 & 13 & 59 & $78,0 \%$ \\
\hline Medio & 10 & 9 & 19 & $52,6 \%$ \\
\hline Arriba & 1 & 0 & 1 & $100,0 \%$ \\
\hline ------------- & ------------- & ------------- & ------------- & ---------- \\
\hline Izquierda & 24 & 11 & 35 & $68,6 \%$ \\
\hline Centro & 6 & 3 & 9 & $66,7 \%$ \\
\hline Derecha & 27 & 8 & 35 & $77,1 \%$ \\
\hline
\end{tabular}

Fuente: Elaboración propia. 
De manera semejante, dadas las diferentes combinaciones de las estrategias posibles para el arquero, los resultados generan la matriz de posibilidades presentada en la tabla 4:

Tabla 4. Resultados de los arqueros

\begin{tabular}{|l|c|c|c|c|}
\hline & Goles & Fallos & Total & \% Gol \\
\hline Izquierda-Arriba & 1 & 0 & 1 & $100,00 \%$ \\
\hline Izquierda-Centro & 4 & 5 & 9 & $44,44 \%$ \\
\hline Izquierda-Abajo & 19 & 6 & 25 & $76,00 \%$ \\
\hline Centro-Arriba & 0 & 0 & 0 & $0 \%$ \\
\hline Centro-Medio & 4 & 2 & 6 & $66,67 \%$ \\
\hline Centro-Abajo & 2 & 1 & 3 & $66,67 \%$ \\
\hline Derecha-Arriba & 0 & 0 & 0 & $0 \%$ \\
\hline Derecha-Medio & 2 & 2 & 4 & $50,00 \%$ \\
\hline Derecha-Abajo & 25 & 6 & 31 & $80,65 \%$ \\
\hline
\end{tabular}

\subsection{Presentación y discusión de resultados}

Con el fin de simplificar el análisis y tener información para cada uno de los escenarios, se definen para el juego tres estrategias para cada uno de los jugadores. Al cobrador se le asignaron para sus lanzamientos dirigidos las estrategias: i) derecha, ii) centro e iii) izquierda. De forma equiparada, para el arquero se le determinaron tres estrategias para atajar el cobro e impedir la anotación: i) derecha, ii) centro e iii) izquierda.

Tabla 5. Resumen de los lanzamientos desde el punto penal

\begin{tabular}{|l|l|c|c|c|c|}
\hline \multicolumn{1}{|c|}{ Jugador } & Arquero & Gol & Fallo & Total & $\%$ Gol \\
\hline Derecha & Derecha & 13 & 3 & 16 & $81,3 \%$ \\
\hline Derecha & Centro & 4 & 0 & 4 & $100,0 \%$ \\
\hline Derecha & Izquierda & 7 & 3 & 10 & $70,0 \%$ \\
\hline Centro & Derecha & 7 & 1 & 8 & $87,5 \%$ \\
\hline
\end{tabular}




\begin{tabular}{|l|l|c|c|c|c|}
\hline \multicolumn{1}{|c|}{ Jugador } & Arquero & Gol & Fallo & Total & \% Gol \\
\hline Centro & Centro & 1 & 3 & 4 & $25,0 \%$ \\
\hline Centro & Izquierda & 5 & 2 & 7 & $71,4 \%$ \\
\hline Izquierda & Derecha & 7 & 4 & 11 & $63,6 \%$ \\
\hline Izquierda & Centro & 1 & 0 & 1 & $100,0 \%$ \\
\hline Izquierda & Izquierda & 12 & 5 & 17 & $70,6 \%$ \\
\hline
\end{tabular}

Organizando la matriz de probabilidades para el juego, se encuentra que existen dos jugadores (cobrador y arquero), cada uno con tres estrategias posibles; para cada combinación de estrategias se asocia una determinada probabilidad. Puesto que los resultados del Maximin y Minimax no coinciden, no existe una solución estable (ver tabla 6).

Tabla 6. Matriz de probabilidades

\begin{tabular}{|c|c|c|c|c|c|}
\hline & \multicolumn{5}{|c|}{ Arquero } \\
\hline & Estrategias & Derecha & Centro & Izquierda & Mínimo \\
\hline \multirow[t]{4}{*}{ Cobrador } & Derecha & 0,813 & 1 & 0,7 & 0,7 \\
\hline & Centro & 0,875 & 0,25 & 0,714 & 0,25 \\
\hline & Izquierda & 0,636 & 1 & 0,706 & 0,636 \\
\hline & Máximo & 0,875 & 1 & 0,714 & \\
\hline
\end{tabular}

Fuente: Elaboración propia.

Si se analiza el juego mediante el uso de Stata, de acuerdo con la evidencia empírica lograda podremos determinar la inexistencia de una solución óptima en estrategias puras en términos del equilibrio de Nash. Como se puede apreciar, el juego no tiene un equilibrio de Nash en estrategias puras ni tampoco bajo la estrategia Maximin, pero, dada la modalidad del juego, se hace necesaria la inclusión de estrategias mixtas; la estructura para los modelos matemáticos lineales por optimizar sigue las estrategias determinadas para el cobrador y el arquero de acuerdo con las formas funcionales siguientes: 
ISSN: 0124-3551 / Año 17, No 27 / julio-diciembre / pp. 297-311

La estructura para el modelo del cobrador tiene la siguiente forma:

$$
\begin{gathered}
\operatorname{Max}^{\prime} \Omega^{`}(5) \\
\text { s.a.: } \\
\Omega^{`} \leq 0,813 \xi_{1}+0,875 \xi_{2}+0,636 \xi_{3} \\
\Omega^{\prime} \leq \xi_{1}+0,25 \xi_{2}+\xi_{3} \\
\Omega^{`} \leq 0,7 \xi_{1}+0,714 \xi_{2}+0,706 \xi_{3} \\
\xi_{1}+\xi_{2}+\xi_{3}=1 \\
\xi_{1}, \xi_{2}, \xi_{3}, \Omega^{`} \geq 0
\end{gathered}
$$

Donde:

' $\Omega$ : Valor del juego del cobrador.

$\xi_{I}$ : Probabilidad del que el cobrador elija efectuar el cobro hacia su derecha.

$\xi_{2}$ : Probabilidad del que el cobrador elija efectuar el cobro hacia su centro.

$\xi_{3}:$ Probabilidad del que el cobrador elija efectuar el cobro hacia su izquierda.

La estructura para el modelo para el arquero tiene la siguiente forma:

$$
\begin{gathered}
\operatorname{Min} \varphi^{\prime}(6) \\
\text { s.a.: } \\
\varphi^{\prime} \geq 0,813 \phi_{1}+\phi_{2}+0,7 \phi_{3} \\
\varphi^{\prime} \geq 0,875 \phi_{1}+0,25 \phi_{2}+0,714 \phi_{3} \\
\varphi^{\prime} \geq 0,636 \phi_{1}+\phi_{2}+0,706 \phi_{3} \\
\phi_{1}+\phi_{2}+\phi_{3}=1 \\
\phi_{1}, \phi_{2}, \phi_{3}, \varphi^{\prime} \geq 0
\end{gathered}
$$

Donde:

$\varphi$ : Valor del juego del arquero.

$\phi_{1}$ : Probabilidad del que el arquero elija cubrir el cobro hacia su derecha.

$\phi_{2}:$ Probabilidad del que el arquero elija cubrir el cobro hacia su centro.

$\phi_{3}:$ Probabilidad del que el arquero elija cubrir el cobro hacia su izquierda. 
La solución a los problemas de optimización planteados dado el problema de maximización al que se enfrenta el cobrador condicionado a sus restricciones asociadas, junto con el problema de minimización al que se ve enfrentado el arquero dadas sus restricciones, da como resultado las probabilidades asociadas con cada una de las estrategias para cada uno de los jugadores junto con el valor esperado de cada una de las estrategias en función de sus respectivas probabilidades (ver tabla 7).

De acuerdo con los hallazgos demostrados, y analizando los resultados conseguidos, se puede afirmar, sin pérdida de generalidad, que, de forma estocástica, en promedio el cobrador debe utilizar la estrategia centro en un $39 \%$ de las veces y utilizar la estrategia de izquierda en un $61 \%$ de las veces que ejecuta un cobro desde el punto penal. Respecto al comportamiento del arquero al momento de la ejecución del cobro, puede afirmarse que, en promedio y de manera estocástica, este debe utilizar la estrategia de lanzarse protegiendo principalmente el centro de la portería en un $1 \%$ de las veces y utilizar la estrategia de proteger su izquierda en un 98,9\% de las veces cuando el cobrador patea desde el punto penal.

De los hechos estilizados encontrados y demostrados en el estudio de las ejecuciones y de los diferentes movimientos realizados por los participantes del juego, se encontró que en la mayoría de las ocasiones no siempre resulta ser así, que tanto el cobrador como el arquero eligen su lado natural para proteger el cobro o para realizar el lanzamiento desde el punto penal.

Tabla 7. Matriz de soluciones para la estrategia mixta

\begin{tabular}{|c|c|c|c|c|c|c|c|}
\hline & & & & & Arquero & & \\
\hline & & & $\operatorname{Min} \phi$ & Prob. 1 & Prob. 2 & Prob. 3 & Sum. Prob. \\
\hline & & & 0,7091029 & 0 & 0,01055409 & 0,98944591 & 1 \\
\hline & $\operatorname{Max} \Omega$ & 0,7091029 & Estrategias & Derecha & Centro & Izquierda & $\begin{array}{c}\text { Valor } \\
\text { Esperado }\end{array}$ \\
\hline Cobrador & Prob. 1 & 0 & Derecha & 0,813 & 1 & 0,7 & 0,703166227 \\
\hline & Prob. 2 & 0,3878628 & Centro & 0,875 & 0,25 & 0,714 & 0,709102902 \\
\hline & Prob. 3 & 0,6121372 & Izquierda & 0,636 & 1 & 0,706 & 0,709102902 \\
\hline & $\begin{array}{l}\text { Sum. } \\
\text { Prob. }\end{array}$ & 1 & $\begin{array}{c}\text { Valor } \\
\text { Esperado }\end{array}$ & 0,72869921 & 0,7091029 & 0,7091029 & \\
\hline
\end{tabular}

Fuente: Elaboración propia. 
Un análisis complementario usando la inducción retrospectiva implementada en Stata confirma varios de los resultados y agrega una serie adicional de hechos asociados con los incentivos, el equilibrio, las ideas vinculadas a la incertidumbre y el riesgo. Los resultados de la tabla 8 revelan que el juego no tiene un equilibrio de Nash en estrategias puras, tampoco bajo la estrategia Maximin. El criterio de inducción retrospectiva indica una senda de equilibrio en derecha-derecha. La inducción en retrospectiva reduce la eventualidad de que se susciten variedad de resultados cuando se ignoran las probabilidades, pero persiste el riesgo asociado con la toma de decisión en el cobro del tiro penal.

Tabla 8. Matriz de pagos

\begin{tabular}{|l|c|c|c|}
\hline & \multicolumn{3}{|c|}{ Arquero } \\
\hline Cobrador & Derecha & Centro & Izquierda \\
\hline Derecha & $(.813 ;-.813)$ & $(1 ;-1)$ & $(7 ;-7)$ \\
\hline Centro & $(.875 ;-.875)$ & $(.25 ;-.25)$ & $(.714 ;-.714)$ \\
\hline Izquierda & $(.636 ;-.636)$ & $(1 ;-1)$ & $(.706 ;-.706)$ \\
\hline
\end{tabular}

Fuente: Elaboración propia.

\section{Conclusiones}

Los cobros desde el punto penal definen partidos, clasificaciones y quizás lo más importante en la solución estratégica desde el enfoque de la teoría de juegos, títulos y, con ello, la asignación de campeonatos. En este trabajo se pone de manifiesto cómo la teoría económica en los deportes, y particularmente en el futbol, se pueden relacionar. Para poder evaluar la teoría de juegos en el fútbol usamos un juego simultáneo entre el arquero y el cobrador como un juego de suma cero en presencia de estrategias mixtas.

El trabajo advierte cómo los participantes del juego deben actuar en presencia de circunstancias estratégicas que implican las denominadas estrategias mixtas, en las que un agente elije entre múltiples estrategias diferenciadas, cuando lo que resulta óptimo es no preferir siempre la misma alternativa; esto permite analizar las diferentes interacciones estratégicas humanas en contextos particulares.

Los resultados también permitieron mostrar que los pronósticos y hallazgos encontrados, conjugados con la teoría económica aplicada en la teoría de juegos, funcionan relativamente bien según las probabilidades estadísticas y los valores esperados. En principio, los jugadores realizan su lanzamiento de forma estocástica, pero, a medida que se van conociendo algunos de los resultados, la incertidumbre y el riesgo tienden a disminuir, 
no obstante, es improbable predecir el futuro en la secuencia de los lanzamientos desde el punto penal. Igualmente, la tendencia muestra que el equipo que inicia los cobros tiene cierta ventaja porque existen resultados que reducen la incertidumbre y aumentan la probabilidad de acierto. El análisis retrospectivo muestra cierta predisposición a que ciertos resultados pueden ser replicados, pero siempre existirá un grado de incertidumbre y riesgo asociado con el lanzamiento, más aún cuando el estado emocional juega un papel preponderante en situaciones competitivas.

\section{Referencias}

Apesteguia, J., \& Palacios-Huerta, I (2010). Psychological Pressure in Competitive Environments: Evidence from a Randomized Natural Experiment. American Economic Review, 100(5), 2548-2564.

Chiappori, P., Levitt, S., \& Groseclose, T. (2002). Testing Mixed Strategy Equilibrium When Players Are Heterogeneous: The case of penalty kicks in soccer. American Economic Reviere, 92(4), 1138-1151.

Gibbons, R. (1992). Game Theory for Applied Economists. Princeton: Princeton University Press.

Kreps, D. (1990). A Course in Microeconomic Theory. Princeton: Princeton University Press.

Nash, J. (1950). Equilibrium Points in n-person Games. Proceedings of the National Academy of Sciences 36(1), 48-49.

Palacios-Huerta, I. (2014). Beautiful Game Theory: How Soccer Can Help Economics. Princeton: Princeton University Press.

Palacios-Huerta, I. (2003). Professionals Play Minimax. Review of Economic Studies, 70(2), 395-415.

Palacios-Huerta, I., \& Volij, O. (2008). Experientia Docet: Professionals Play Minimax in Laboratory Experiments. Econometrica, 76(1), 71-115. 
